\title{
Prospective target assessment and multimodal prediction of survival for personalized and risk-adapted treatment strategies in multiple myeloma in the GMMG-MM5 multicenter trial
}

Dirk Hose ${ }^{1,2+}$, Susanne Beck ${ }^{1,2+}$, Hans Salwender ${ }^{3}$, Martina Emde ${ }^{1,2}$, Uta Bertsch², Christina Kunz ${ }^{4}$, Christoph Scheid ${ }^{5}$, Mathias Hänel ${ }^{6}$, Katja Weisel ${ }^{7}$, Thomas Hielscher ${ }^{4}$, Marc S. Raab², Hartmut Goldschmidt ${ }^{2,8}$, Anna Jauch ${ }^{9}$, Jérôme Moreaux ${ }^{10,11}$ and Anja Seckinger ${ }^{1,2^{*}}$

\begin{abstract}
Background: Personalized and risk-adapted treatment strategies in multiple myeloma prerequisite feasibility of prospective assessment, reporting of targets, and prediction of survival probability in clinical routine. Our aim was first to set up and prospectively test our experimental and analysis strategy to perform advanced molecular diagnostics, i.e., interphase fluorescence in-situ hybridization (iFISH) in $\geq 90 \%$ and gene expression profiling (GEP) in $\geq 80 \%$ of patients within the first cycle of induction chemotherapy in a phase III trial, seen as prerequisite for target expression-based personalized treatment strategies. Secondly, whether the assessment of risk based on the integration of clinical, cytogenetic, and expression-based parameters ("metascoring") is possible in this setting and superior to the use of single prognostic factors.

Methods: We prospectively performed plasma cell purification, GEP using DNA-microarrays, and iFISH within our randomized multicenter GMMG-MM5-trial recruiting 604 patients between July 2010 and November 2013. Patient data were analyzed using our published gene expression report (GEP-R): after quality and identity control, integrated risk assessment (HM metascore) and targets were reported in clinical routine as pdf-document.

(Continued on next page)
\end{abstract}

\footnotetext{
* Correspondence: anja.seckinger@med.uni-heidelberg.de

${ }^{\dagger}$ Dirk Hose and Susanne Beck contributed equally to this work.

${ }^{1}$ Labor für Myelomforschung, Universitätsklinikum Heidelberg, Heidelberg, Germany

${ }^{2}$ Medizinische Klinik V, Universitätsklinikum Heidelberg, Heidelberg, Germany

Full list of author information is available at the end of the article
}

(c) The Author(s). 2019 Open Access This article is distributed under the terms of the Creative Commons Attribution 4.0 International License (http://creativecommons.org/licenses/by/4.0/), which permits unrestricted use, distribution, and reproduction in any medium, provided you give appropriate credit to the original author(s) and the source, provide a link to the Creative Commons license, and indicate if changes were made. The Creative Commons Public Domain Dedication waiver (http://creativecommons.org/publicdomain/zero/1.0/) applies to the data made available in this article, unless otherwise stated. 
(Continued from previous page)

Results: Bone marrow aspirates were obtained from 573/604 patients (95\%) and could be CD138-purified in 559/573 (97.6\%). Of these, iFISH-analysis was possible in 556 (99.5\%), GEP in 458 (82\%). Identity control using predictors for sex, light and heavy chain type allowed the exclusion of potential sample interchanges (none occurred). All samples passed quality control. As exemplary targets, IGF1R-expression was reported expressed in 33.1\%, AURKA in 43.2\% of patients. Risk stratification using an integrated approach, i.e., HM metascore, delineated 10/77/13\% of patients as high/medium/ low risk, transmitting into significantly different median progression-free survival (PFS) of 15 vs. 39 months vs. not reached (NR; $P<0.001)$ and median overall survival $(O S)$ of 41 months vs. NR vs. NR $(P<0.001)$. Five-year PFS and OSrates were $5 / 31 / 54 \%$ and $25 / 68 / 98 \%$, respectively. Survival prediction by HM metascore (Brier score $0.132, P<0.001$ ) is superior compared with the current gold standard, i.e., revised ISS score $(0.137, P=0.005)$.

Conclusions: Prospective assessment and reporting of targets and risk by GEP-R in clinical routine are feasible in $\geq 80 \%$ of patients within the first cycle of induction chemotherapy, simultaneously allowing superior survival prediction.

Keywords: Multiple myeloma, Risk, Metascoring, Reporting, Survival

\section{Background}

Multiple myeloma is a malignant hematological disease characterized by accumulation of clonal plasma cells in the bone marrow and associated clinical signs and symptoms, especially those related to the displacement of normal hematopoiesis and generation of osteolytic bone disease [1].

Introduction of high-dose melphalan followed by autologous stem cell transplantation, proteasome inhibitors, immunomodulatory drugs, and monoclonal antibodies improved survival [2-5] without curing a significant fraction of patients. Prognosis of individual patients is highly heterogeneous: current treatment algorithms do well for some patient groups with a median survival of more than 10 years, while a significant proportion of patients shows median survival of 2 years or even below [6, 7]. This frequently prompts the suggestion to treat the latter patients differently, most often more aggressively, in other terms to perform risk-adapted treatment, e.g., UAMS total therapy [8], mSMART by the Mayo Clinic [9], or the GMMGCONCEPT trial (NCT03104842).

Risk stratifications in clinical routine are usually performed by combination of presence of high-risk chromosomal aberrations as detected by interphase fluorescence in-situ hybridization (iFISH) and the International Staging System (ISS) as exemplified by the revised ISS score (rISS) and others [10-12]. At the same time, prognostic power can be increased by assessing gene expression, i.e., proliferation [13] and high risk scores [14-20], e.g., by DNAmicroarrays (gene expression profiling, GEP). This is, however, rarely used prospectively in clinical routine or in a clinical trial setting. In turn, ending up with a variety of clinical and molecular prognostic factors, it is necessary to integrate different factors into a single prognostic information (metascoring).

Besides risk stratification, assessment of gene expression allows investigation of aberrantly or overexpressed targets exemplified by Aurora-kinase A (AURKA) [21] or insulin- like growth factor 1 receptor (IGF1R) [22]. Expression of these targets, in turn, is associated with adverse survival $[21,22]$. For potential personalized treatment, the addition of clinical grade inhibitors to a backbone treatment only in those patients whose myeloma cells actually express the respective target could be envisioned. Whereas the number of actionable targets in multiple myeloma is currently largely limited, it is very likely that the advent of immunotherapy will change this.

Both personalized and risk-adapted treatment strategies prerequisite the feasibility of prospective assessment and reporting of targets and prediction of survival probability in clinical routine in a high enough percentage of patients. For phase III trial strategies, e.g., selecting an add-on treatment, the necessary threshold based on power calculations and clinical feasibility could be estimated as $80 \%$ of the actual population of patients included in the trial. It is interesting to denote that, despite of course iFISH and GEP have been used in an academic setting or using commercial providers $[8,17,23-28]$, the question has yet not been answered if this is possible in terms of a prospective molecular analysis and reporting, as opposed to being conducted in a prospective clinical trial. Our study group sees such a proof as prerequisite for indeed planning a clinical trial based on molecular diagnostics such as GEP or RNA-sequencing.

Aims of this study were thus: (i) set up a sampling, experimental and analysis strategy to perform iFISH in $\geq 90 \%$ and GEP in $\geq 80 \%$ of patients within the first cycle of induction chemotherapy. (ii) Report to patients and physicians within this time to be able to draw a clinical consequence. (iii) Prospectively validate this strategy in the randomized phase III multicenter GMMG-MM5-trial including assessment of potential targets (based on GEP) and multimodal assessment of risk using clinical, cytogenetic, and gene expression-based prognostic factors and their integration into a metascore in clinical routine. 


\section{Methods}

\section{Patients}

Six hundred and four patients were included in the prospective, open-label, randomized multicenter phase III clinical trial (EudraCT no. 2010-019173-16) between July 2010 and November 2013. A total of 31 transplant centers and 75 associated sites throughout Germany are participating in this trial initiated by the GMMG and approved by ethics committees of the University of Heidelberg and all participating sites. The MM5 trial was conducted according to the European Clinical Trial Directive (2005) and the Declaration of Helsinki. Patients were equally randomized to each of the four treatment arms (A1, A2, B1, and B2) using block randomization, stratified by ISS stage. Treatment consisted of either three 4-week cycles of PAd $(\mathrm{A} 1+\mathrm{B} 1)$ or three 3-week cycles of VCD (A2+B2). Thereafter, standard intensification according to local protocols (GMMG standard) was performed, including stem cell mobilization and leukapheresis followed by single high-dose therapy or, for patients not achieving near complete response (nCR) or better, tandem high-dose therapy. Subsequently, consolidation therapy consisting of two cycles of lenalidomide (25 mg, days 1-21) followed by lenalidomide maintenance (for the first 3 months, $10 \mathrm{mg} /$ day continuously and thereafter $15 \mathrm{mg} /$ day continuously) for either 2 years $(A 1+A 2)$ or until CR $(B 1+B 2)$ was applied. Patients were followed until April 2017. Trial results regarding the primary endpoint, i.e., non-inferiority of VCD vs. PAd induction treatment have already been published [29], or are currently under review. With written informed consent as depicted above, patients were simultaneously included in the pre-planned prospective conducting of advanced molecular diagnostics, i.e., iFISH and GEP.

\section{Sampling strategy}

Sixty to $80 \mathrm{ml}$ of heparinized bone marrow (i.e., $3-4 \times$ $18 \mathrm{ml}$ of bone marrow plus $2 \mathrm{ml}$ of heparin per syringe, aspirated with three bone marrow punctures within one anesthetized region) were drawn before the start of chemotherapy and sent to the Multiple Myeloma Research Laboratory Heidelberg by overnight express. For in-house samples, technicians of the Multiple Myeloma Research Laboratory attended the bone marrow aspiration providing assistance to the physician and allowing the fastest possible processing of the samples. Plasma cell purification including quality control and preparation of samples for iFISH (i.e., cytospins) and GEP (see below for details) were performed centrally according to our laboratory SOP immediately after the arrival of the sample. Sample submitting centers were informed electronically about the quality of the aspirate and the results of the plasma cell purification.

\section{Purification of $\mathrm{CD}_{138^{+}}$plasma cells}

Density gradient centrifugation of bone marrow aspirates over Ficoll Hypaque (Biochrom, Berlin, Germany) was performed to separate mononuclear cells by standard protocol. CD $138^{+}$plasma cells were isolated using anti-CD138 immunobeads and an autoMACS Pro Separator (Miltenyi Biotec, Bergisch Gladbach, Germany) as published [13, 21, 30-35]. Purity was assessed by flow cytometry (Becton Dickinson, Heidelberg, Germany) using antibodies against CD38 (clone HB-7, FITClabeled; Becton Dickinson) and CD138 (clone B-B4, PElabeled; Miltenyi Biotec). Aliquots of $\mathrm{CD} 138^{+}$malignant plasma cells were subjected to cytospin preparation with 5000 cells per dot for iFISH analysis $(n=556$ patients) and RNA/DNA extraction for gene expression profiling $(n=458)$.

\section{Interphase fluorescence in situ hybridization}

iFISH analysis was conducted on CD138-purified plasma cells using probes for numerical changes of the chromosome regions $1 \mathrm{q} 21,5 \mathrm{p} 15,5 \mathrm{q} 31$ or $5 \mathrm{q} 35,8 \mathrm{p} 21,9 \mathrm{q} 34$, $11 \mathrm{q} 22.3$ or $11 \mathrm{q} 23,13 \mathrm{q} 14.3,15 \mathrm{q} 22,17 \mathrm{p} 13$, and $19 \mathrm{q} 13$, as well as translocations $\mathrm{t}(4 ; 14)(\mathrm{p} 16.3 ; \mathrm{q} 32.3), \mathrm{t}(11 ; 14)(\mathrm{q} 13$; $\mathrm{q} 32.3)$, and $\mathrm{t}(14 ; 16)(\mathrm{q} 32.3 ; \mathrm{q} 23)$ or any other IgH rearrangement with unknown translocation partner, according to the manufacturer's instructions (Kreatech, Amsterdam, The Netherlands and MetaSystems, Altlussheim, Germany) and data were analyzed as published [36].

\section{Analysis of gene expression}

RNA was extracted using the Qiagen AllPrep DNA/ RNA kit (Qiagen, Hilden, Germany) according to the manufacturer's instructions. Quality control and quantification of total RNA was performed using an Agilent 2100 bioanalyzer (Agilent, Frankfurt, Germany).

Gene expression profiling using U133 2.0 plus arrays (Affymetrix, Santa Clara, CA, USA) was performed as published [13, 32, 33]. Expression data are deposited in ArrayExpress under accession number E-MTAB-2299.

\section{Reporting of GEP-R}

Our gene expression report (GEP-R) [31] is a noncommercial software framework developed within the open source software environments $R$ [37] and Bioconductor [38] that can be adapted to other parameters or disease entities. It includes classifications of myeloma, i.e., TC [39]-, EC [40]-, and molecular classification [41], risk stratification, i.e., UAMS GEP70 [14]- and IFM 15gene score [15], and our gene expression-based proliferation index (GPI) [13], and assessment of target gene expression, e.g., for immunotherapeutic or individualized treatment approaches, into one report. The GEP-R runs a quality and identity control; the latter is based on prediction analysis for microarrays (PAM) [42] predictors 
for sex, IgL (lambda, kappa), and IgH type (IgA, IgG, IgD). Results are reported as pdf document consisting of a two pages report given to the treating physician and an appendix containing details regarding the quality and identity control, as well as the assessment of gene expression. Within the GEP-R, the implemented HM metascore integrates gene expression-based and conventional prognostic factors into one prognostic classification [31]. The HM metascore has already been validated by crossvalidation and an external validation cohort [31].

\section{Statistical analysis}

Effects were considered statistically significant if the Pvalue of corresponding statistical tests was below $5 \%$. Overall (OS) and progression-free survival (PFS) were investigated using Cox's proportional hazard model as published [43,44]. Survival curves were computed with nonparametric survival estimates for censored data using the Kaplan-Meier method [45, 46]. Difference between the curves was tested using the G-rho Log-rank test [47]. A subset of 451 patients with complete prediction information was used within a 73 months period for evaluating the performance of the risk prediction models in OS analysis. Cox's proportional hazard models were used as input; for cross-validation, subsampling parameter of 301 and bootstrapping parameter of 150 were chosen. The integrated Brier score was used to assess prediction accuracy [48-50]. For statistical testing, the van de Wiel test was used [50].

\section{Results}

\section{Feasibility of sampling and plasma cell purification}

Six hundred and four patients were included in the GMMG multicenter MM5-trial between July 2010 and November 2013, with a total of 31 participating transplant centers and 75 associated sites throughout Germany. In accordance with the pre-planned prospective protocol, bone marrow aspirates at the time of inclusion in the study, i.e., before start of treatment, were available for $n=573$ patients (94.9\%) with a median volume of $75 \mathrm{ml}$ (standard deviation (SD): 23.4), of whom we were able to successfully perform plasma cell purification followed by quality control using flow cytometry for $n=559$ patients (97.6\%) according to our laboratory SOP. The 31 lacking samples $(5.1 \%)$ were due to patients declining the bone marrow aspiration (2.5\%) or punctio sicca (2.5\%) (Fig. 1). Median purity according to CD38/ CD138 double staining was $87.9 \%$ (SD 16.5\%) with a median cell number of $1.2 \times 10^{6}$ cells (SD $\left.8.5 \times 10^{6}\right)$.

\section{Feasibility of iFISH and GEP}

iFISH using cytospins from CD138-purified plasma cells was performed centrally for the trial (Multiple Myeloma Research Laboratory and Department of Human Genetics).
Cytospins were prepared with 5000 cells per dot on the day of purification and hybridized immediately thereafter. Data could be obtained for 556/573 patients with available bone marrow aspirates (97\%) and 556/559 patients with available CD138-purified plasma cells, respectively (99.5\%; Fig. 1). The median proportion of malignant plasma cells as per iFISH, i.e., the highest percentage of a chromosomal aberration, was 95\% (SD 20\%).

Samples for RNA extraction followed by quality control were collected over 2 weeks each and then subjected to gene expression profiling by DNA-microarrays. In total, $n=458$ transcriptome datasets are available; i.e., $81.9 \%$ of patients with available CD138-purified plasma cells. Of these, two patients were excluded from further analysis as they did not fulfill the inclusion criteria of the trial. Gene expression profiling could not be performed in 53 cases due to low RNA quality (9.5\%) and further 48 cases $(8.6 \%)$ in which not enough RNA was available (Fig. 1). Gene expression data were then analyzed and reported using our previously published GEP-R [31].

\section{Identity and quality control}

In the 456 GEP-R from the intention-to-treat population, we exclude an interchange of samples using the implemented identity control. Predictors for light and heavy chain type (overall error rate $2.2 \%$ and $1.6 \%$, respectively) as well as the sex of the patient (5.5\% overall error rate, in agreement with frequent loss of the Ychromosome [51]; being "female" can be predicted without error) showed comparable results in this prospectively analyzed cohort of patients if compared to the original publication (retrospectively on the validation cohort: $6 \%, 1 \%$, and $4 \%$, respectively [31]). False predictions were found in $3 / 93$ patients with IgA myeloma (3.2\%) and $3 / 269$ patients with IgG (1\%) as well as $4 / 308$ patients with kappa light chains (1.3\%) and 6/148 patients with light chains type lambda (4\%; Additional file 1: Table S1). No sample failed in all three criteria.

As per the implemented quality control with a total of 7 quality parameters, the majority of patients showed no abnormality $(339,74.4 \%), 110$ had a warning in one minor criterion (24.1\%), 6 patients in $2(1.3 \%)$, and 1 patient in 3 $(0.2 \%)$. No patient fulfilled one of the major exclusion criteria based on previously performed QC analysis.

\section{Risk assessment and classifications of myeloma}

Fifty-three patients were predicted using the GEP-R to have a $t(4 ; 14)$, corresponding to $11.6 \%$ of the total cohort with available GEP data. In five patients, iFISH and GEP showed discrepant results with the alteration being not found in iFISH. In conservative estimation, we considered the PAM-based predictor to have an overall error rate of $1.1 \%$. Three of these patients had a clonal IgHtranslocation with an unknown translocation partner, 


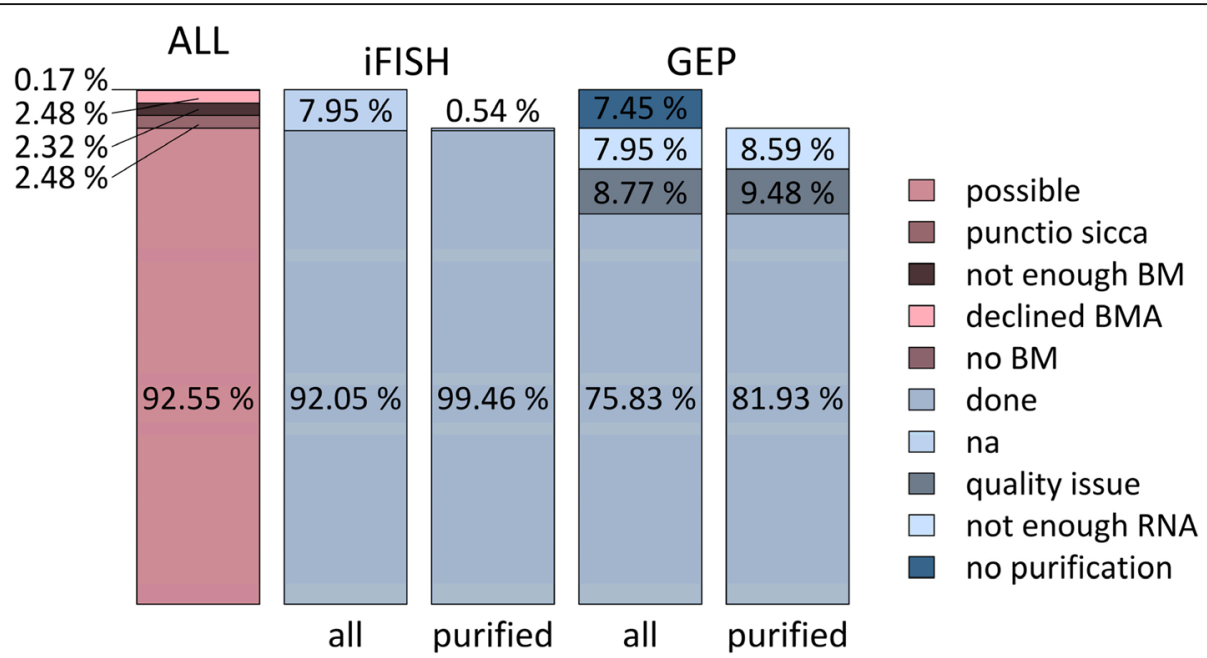

Fig. 1 Feasibility of plasma cell purification, iFISH, and gene expression profiling within the GMMG-MM5 trial. Percentages are given for feasibility of plasma cell purification (ALL) as well as performing of interphase fluorescence in situ hybridization (iFISH) and gene expression profiling (GEP) using DNA-microarrays. For the latter two, columns refer to both all patients and those for which purified plasma cells were available. BM(A), bone marrow (aspiration); na, not available

one patient had a clonal $\mathrm{t}(11 ; 14)$, and one patient did not have a detectable translocation involving the IgHlocus. Predicted $\mathrm{t}(4 ; 14)$ status delineated significantly different median PFS of 40 vs. 26 months $(P=0.008)$ and median OS of not reached (NR) vs. 56 months $(P=$ 0.003; Additional file 2: Figure S1).

Regarding the GEP70 score, 113 patients were attributed as being high risk (24.8\%) and $343(75.2 \%)$ as low risk which transmitted into significantly different median PFS of $23 \mathrm{vs.}$ 43 months $(P<0.001)$ and median OS of 41 months vs. NR $(P<0.001$; Additional file 2: Figure S1). The same holds true for the IFM15 score with 106 patients being high risk (23.2\%) and 350 patients (76.8\%) being low risk transmitting into median PFS of 25 vs. 44 months $(P<0.001)$ and OS of 57 months vs. NR $(P<0.001$; Additional file 2: Figure S1). Regarding myeloma cell proliferation, 36 (7.9\%), 191 (41.9\%), and 229 (50.2\%) patients were attributed as being $\mathrm{GPI}^{\text {high }}, \mathrm{GPI}^{\text {medium }}$, and $\mathrm{GPI}^{\text {low }}$, respectively with significantly different median PFS of 18 vs. 33 vs. 49 months $(P<$
$0.001)$ and median OS of 33 vs. 76 months vs. NR $(P<$ 0.001; Additional file 2: Figure S1).

The percentages of patients identified as being of high risk by conventional prognostic factors, gene expressionbased risk scores or proliferation (GPI), as well as metascores and the overlap of the respective groups of patients are shown in Table 1 and Fig. 2.

For the results of grouping myeloma into different subentities, see Additional file 2: Figure S2.

\section{Target assessment}

Expression height and presence/absence of expression were assessed for (i) examples of potential "target genes." As the aim of our manuscript was prospective testing of the GEP-R, these examples include AURKA, FGFR3, and IGF1R, for which at that time potential clinical grade inhibitors were foreseen, (ii) potential targets for immunotherapy, i.e., cancer testis antigens like CTAG1, MAGE1, and HM1.24, and (iii) genes frequently

Table 1 Delineation of "high-risk" patients by the respective variables and scores

\begin{tabular}{|c|c|c|c|c|c|c|c|c|c|}
\hline & $\begin{array}{c}\% \\
\text { as high risk }\end{array}$ & $\begin{array}{c}\mathrm{HM}- \\
\text { metascore }\end{array}$ & GPI & GEP70 & IFM15 & $\begin{array}{c}\text { predicted } \\
t(4 ; 14)\end{array}$ & ISS & rISS & $\begin{array}{c}\text { None of } \\
\text { others }\end{array}$ \\
\hline HM-metascore & 10.0 & -- & 54.3 & 37.5 & 33.3 & 46.2 & 21.9 & 41.8 & 0 \\
\hline GPI & 7.8 & 42.2 & -- & 22.3 & 25.7 & 5.8 & 14.1 & 21.8 & 0 \\
\hline GEP70 & 24.8 & 93.3 & 71.4 & -- & 43.8 & 76.9 & 31.3 & 47.3 & 22.3 \\
\hline IFM15 & 23.3 & 77.8 & 77.1 & 41.1 & -- & 36.5 & 27.3 & 43.6 & 36.2 \\
\hline predicted $t(4 ; 14)$ & 11.5 & 53.3 & $8, .6$ & 35.7 & 18.1 & -- & 10.9 & 25.5 & 15.4 \\
\hline ISS & 28.4 & 62.2 & 51.4 & 35.7 & 33.3 & 26.9 & -- & 100 & 38.3 \\
\hline rISS & 12.2 & 51.1 & 34.3 & 23.2 & 22.9 & 26.9 & 43.0 & -- & 0 \\
\hline None & 44.1 & -- & -- & -- & -- & -- & -- & -- & -- \\
\hline
\end{tabular}

The percentages of patients identified as being of high risk (first column) and the overlap of the respective groups of patients are shown $G P I$, gene expression-based proliferation index; $(r) / S S$, (revised) International Staging System 


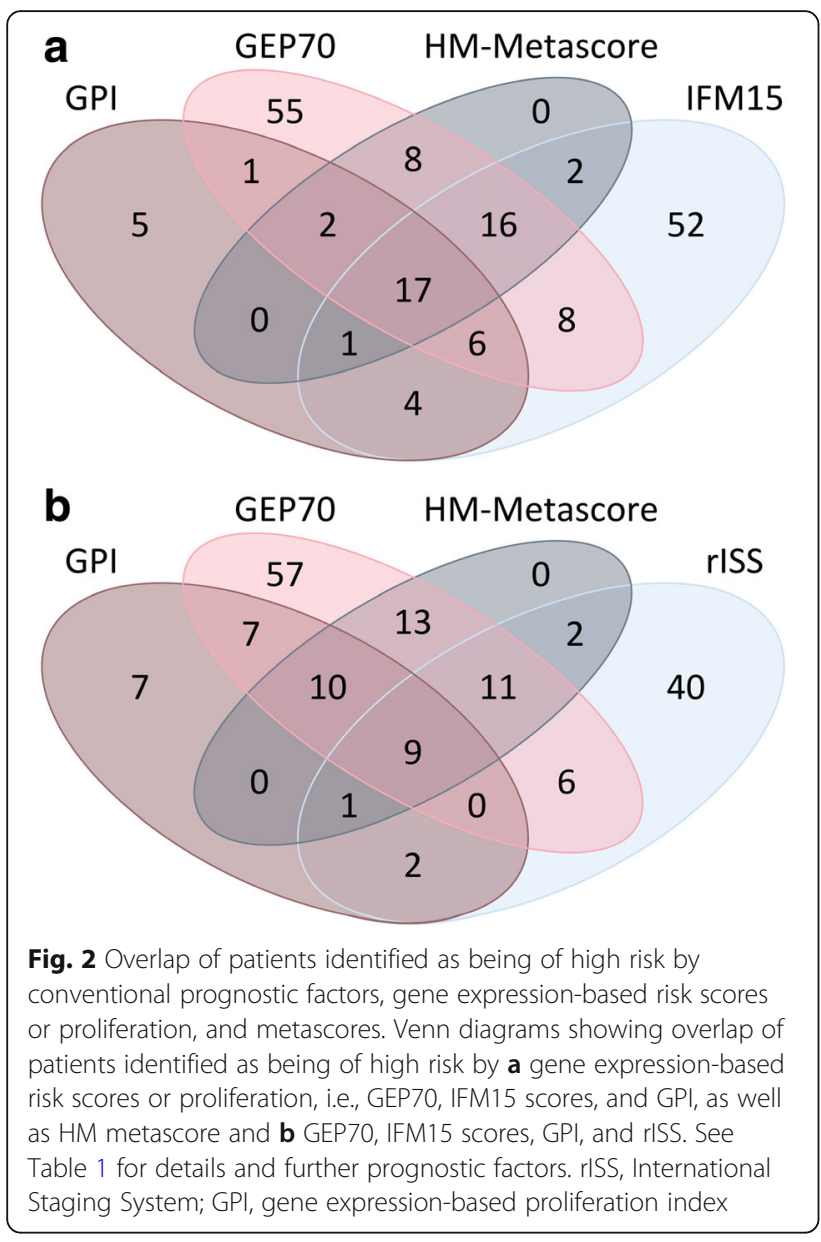

aberrantly or differentially expressed in myeloma. In our cohort of 456 patients from the intention-to-treat population for which a GEP-R is available, 197 were found to express AURKA (43.2\%), 151 IGF1R (33.1\%), and 50 FGFR3 (11\%), respectively. In the same way, candidates for personalized treatment approaches, given the availability of respective inhibitors, could be addressed.

\section{Metascoring}

The HM metascore had already been validated on an external cohort as part of its initial set up [31]. In prospective testing, 58 myeloma patients were classified as being low risk (12.7\%), 352 as medium risk (77.2\%), and 46 were attributed as being high risk (10.1\%). This transmitted into significant different median PFS of NR vs. 39 vs. 15 months $(P<0.001)$ and OS of NR vs. NR vs. 41 months $(P<0.001)$. At 5 years, survival rate was $98 \%$ vs. $68 \%$ vs. $25 \%$ and $57(98.2 \%)$ vs. $253(71.8 \%)$ vs. 15 (32.6\%) patients were still alive (Fig. 3).

Applying the rISS as the current gold standard, 150 patients were classified as being rISS I (27.8\%), 317 as rISS II (58.9\%), and 71 were attributed as rISS III (13.2\%), respectively, transmitting into significant different median PFS of 54 vs. 37 vs. 22 months $(P<$ $0.001)$ and median OS of NR vs. NR vs. 41 months $(P<$ $0.001)$. Five-year PFS and OS-rates for rISS I/II/III were $44 / 30 / 18 \%$ and $86 / 65 / 40 \%$, respectively (Fig. 3). Forest plots summarizing the prognostic impact of individual factors and metascores (i.e., rISS and HM metascore) can be found in Fig. 4.

An integrated Brier score was calculated to assess prediction accuracy. The overall predictive value for OS from 0 to 73 months is best for the $\mathrm{HM}$ metascore (0.132) compared to rISS (0.137) or other GEP-based scores, i.e., the GEP70- (0.139), IFM15 models (0.14), or Reference (0.148), respectively (Fig. 5). Differences between the HM metascore and the other models were, however, not statistically significant. Likewise, differences between the "conventional" ISS and rISS were not significantly different.

\section{Discussion}

Personalized and risk-adapted treatment strategies in multiple myeloma prerequisite the feasibility of prospective assessment, reporting of targets and prediction of survival probability in clinical routine-in a "high enough" percentage of patients. Besides, they also necessitate the availability of treatment options that can indeed be applied in a personalized manner (see below). Based on statistical power calculations for subsequent trials, we defined the thresholds as $90 \%$ for iFISH and $80 \%$ for GEP-based assessment.

\section{Can you do it in clinical routine?}

Despite iFISH, GEP and RNA-sequencing have been used in academic setting or using commercial providers, to the best of our knowledge, no data is available regarding a prospective molecular analysis and reporting in clinical routine in a way that this could be used for personalized and risk-adapted treatment strategies, e.g., during the first cycle of induction chemotherapy. This is a different setting from being able to run advanced molecular diagnostics in a prospective trial in a subpopulation of patients which has frequently been shown by others and us [8, 17, 23-28].

In our GMMG-MM5 trial, consenting to bone marrow aspiration and molecular analyses was not a prerequisite to participate in the trial, in contrast, e.g., to the total therapy 4 and 5 trial with mandatory GEP data $[8,23]$, as it did not imply any up-front clinical consequences. Nonetheless, $95 \%$ of patients agreed to bone marrow aspiration at inclusion in the trial. This rate was driven by a high motivation of participating centers and physicians to explain patients planned analyses and the usefulness of translational research in multiple myeloma, and a concomitant intrinsic willingness of patients to participate. At the same time, we motivated participating 


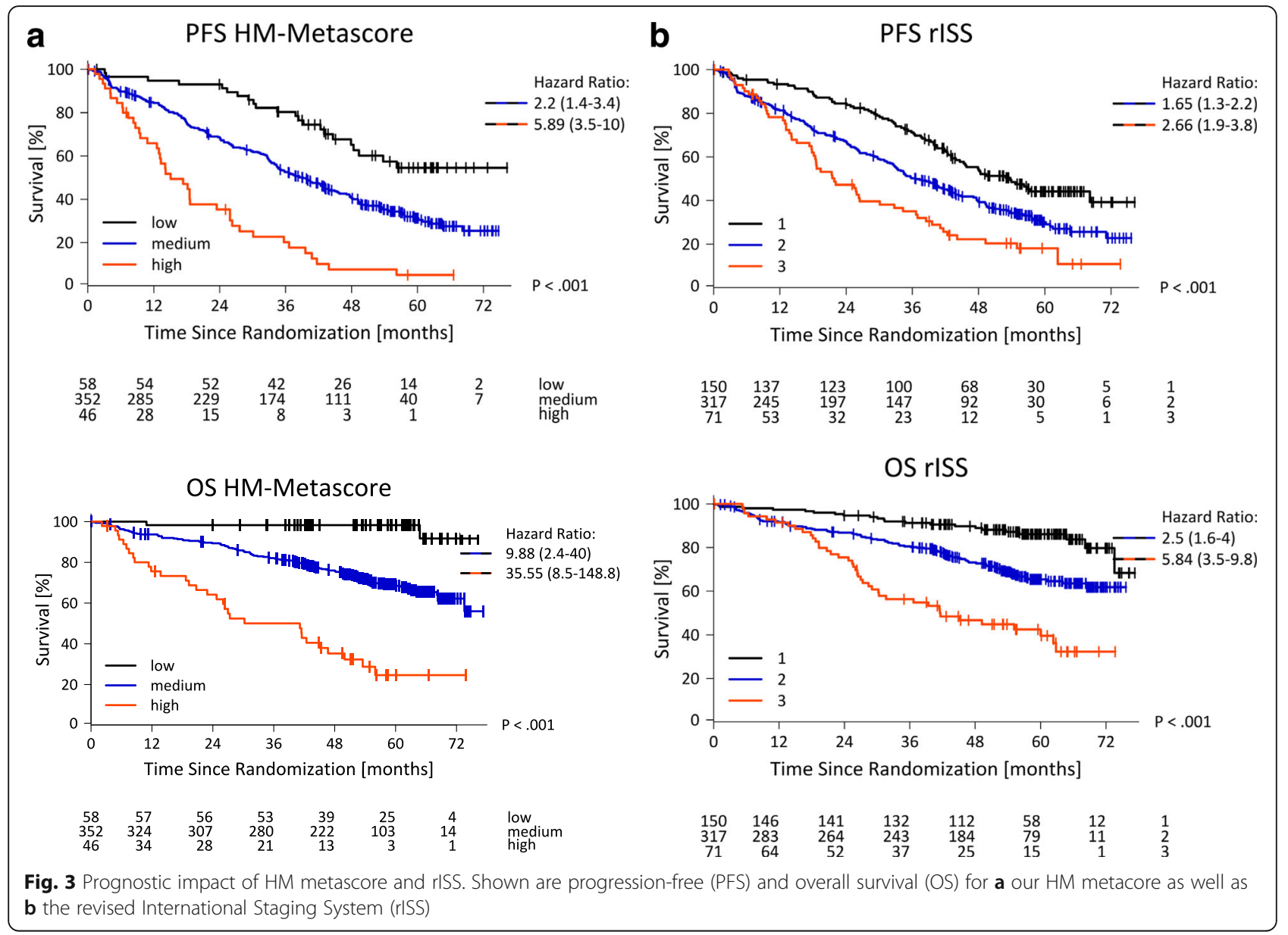

centers by direct feedback of sample quality and result of purification, as well as iFISH and GEP (see below). The set-up of our sampling and analysis strategy allowed performing iFISH in $97 \%$ and GEP in $80 \%$ of patients with available bone marrow samples. This compares favorably to iFISH results reported by the EMN02/HOVON95-trial (74.1\%) [52], IFM2009-trial (73.6\%) [53], DSMM XI-trial (73.7\%) [54], SWOG S0777-trial (60.2\%) [55], or a pooled analysis of three PETHEMA/GEM clinical trials, i.e., GEM2000, GEM2005MENOS65, and GEM2010MAS65 $(60.8 \%)$ [56], which were conducted during a comparable time frame. We have therefore for the first time validated prospectively in a randomized phase III multicenter trial the possibility to perform not only cytogenetic (including rISS) but also gene expression-based risk stratification and reporting in $>80 \%$ of patients during the first cycle of induction chemotherapy as-potentially-molecular riskadapted, personalized treatment strategy.

\section{Why would you want to do it?}

Besides risk stratification which can be done by both iFISH and GEP [20], the specific benefit of gene expression profiling, either by DNA-microarrays as in this trial or by RNA-sequencing, lies in the additional ability to identify target gene expression. In our analysis, this was intended for immunological targets and those for which small molecules or antibodies existed, e.g., Aurorakinase A (VX-680 [21]), IGF1-receptor (e.g., AVE1642 [57]), or FGF3R (e.g., CHIR-258 [58]). AURKA was selected at this point in time when the GEP-R was developed as we had previously shown it to be expressed in approx. $30 \%$ of previously untreated myeloma patients and is associated with adverse survival [21]. IGF1R-inhibition was selected due to its importance as myeloma growth factor and impact on patient survival $[22,59]$. As our aim was the prospective testing of our approach, we retained both factors also in the HM metascore, even given that neither will in 2019 be used as a clinical target. Here, it was our intention to give the proof-of-principle for prospective advanced molecular diagnostics of targets and reporting in clinical routine; in this way, the GEP-R is depicted and should be interpreted. Novel targets for which clinical grade inhibitors become available or immunological targets can be added to the assessment due to the adaptable surface of our reporting tool (GEP-R) [31]. Without a doubt, actual implementation necessitates 


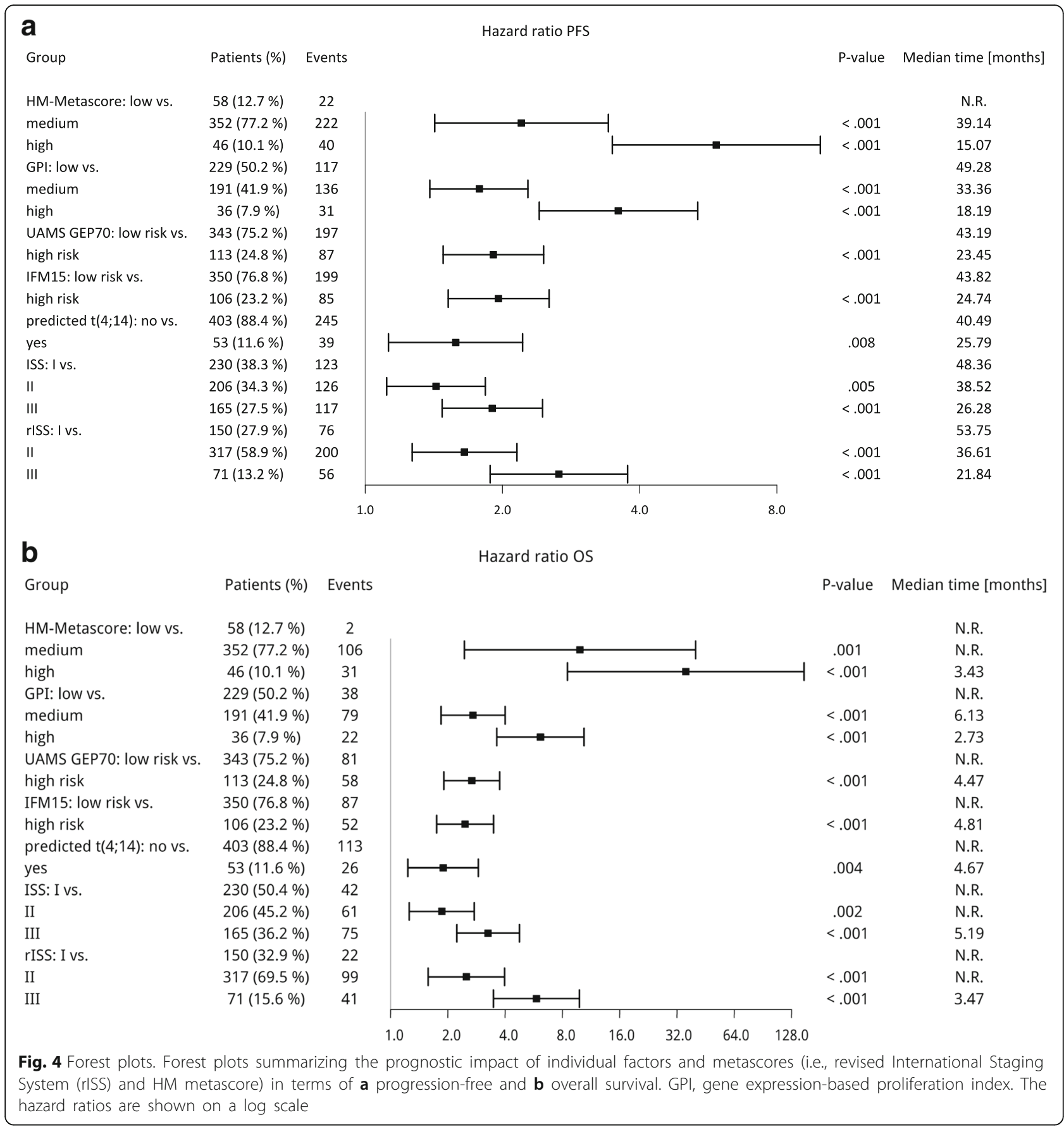

standardization and either commercial or academic development of an actual molecular diagnostics test. Although this is beyond the scope of our manuscript, we show here that such a strategy is in principle feasible.

Alongside the principle possibility of running advanced molecular profiling and depiction of potential targets for individualized treatment, assessment of risk was the third main objective of our study. Here, many prognostic factors have been described with the ongoing discussion of which to include $[19,20]$.
This leaves the treating physician with a plethora of information that is difficult to consolidate intending counseling patients and drawing a clinical conclusion. Metascoring appears as an appropriate strategy $[18,31,60,61]$ to overcome this, and we show that this is likewise possible in a randomized clinical trial setting. Regarding the molecular techniques used in the metascore, i.e., iFISH and GEP, we choose to include both due to in part non-overlapping prognostic information, e.g., it is not possible to predict del17p13 at a high-enough accuracy by GEP [62] 

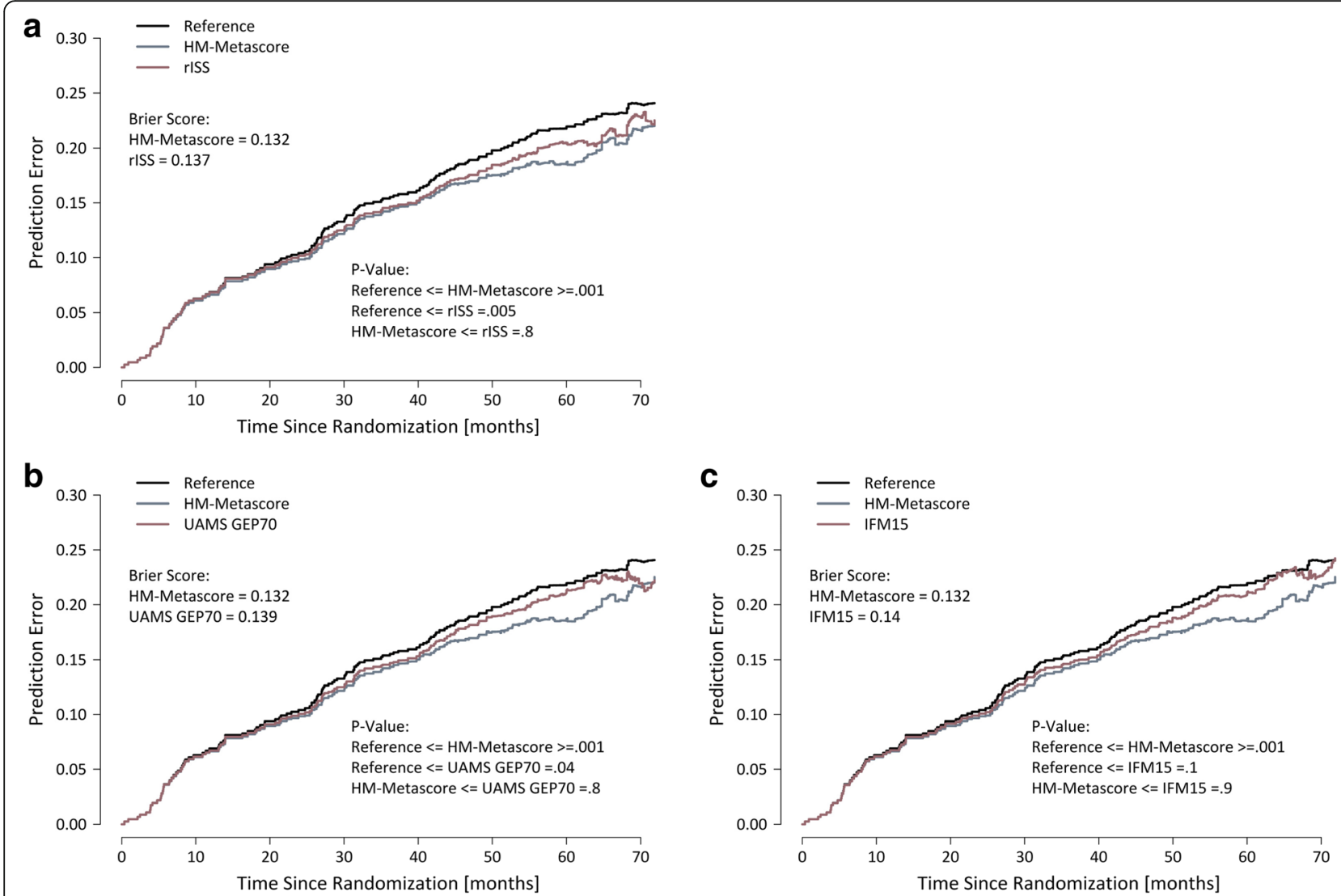

Fig. 5 Brier score. Integrated Brier score assessing accuracy of prediction for HM metascore vs. a revised International Staging System (rISS), b GEP70 score, and c IFM15 score compared to the reference. Brier scores (prediction error) as well as $P$ values for the different comparisons are given (bottom right, respectively) for overall survival are given

The actual (good) prediction result of our metascore per se is thereby not the main focus of our analysis. Nonetheless, even with this "2010 choice" regarding risk factors and target genes, metascoring including GEPbased risk assessment is superior in numbers to rISS, although not statistically so. The same however holds true for a comparison of rISS to ISS even on our comparably large cohort of patients.

\section{Can we do better?}

For a molecular diagnostics-based trial, bone marrow assessment, submission of bone marrow of sufficient quality and quantity are mandatory prerequisites. Based on our optimized protocol, data in terms of purity and cell number could be further improved within the prospective multicenter GMMG-HD6 trial (NCT02495922) recruiting 564 patients between June 2015 and September 2017 to a median purity of $94.5 \%$ (SD 13.2\%) and 1.4 $\times 10^{6}\left(\mathrm{SD} 34.8 \times 10^{6}\right)$ purified plasma cells. Future directions comprise the optimization of patient recruitment and sampling strategy in terms of mandatory bone marrow aspiration as inclusion criterion as defined above and replacement of GEP by RNA-sequencing, currently tested in our BMBF-funded CLIOMMICS-project. RNAsequencing has several advantages over GEP using microarrays [63, 64]: (i) it provides quantification of levels of transcripts without significant saturation effects, (ii) it does not prerequisite a priori definition of sequences to be analyzed (as are, e.g., Affymetrix "probesets") and thus allows detection of mutated transcripts, e.g., targetable BRAF-mutations. Likewise, transcripts, for which initially incorrect sequences were assumed, and thus corresponding probesets do not interrogate the transcript of interest, can be analyzed. (iii) RNA-sequencing enables the analysis of splice variants, as well as (iv) the investigation of other RNA types, for example, miRNAs [65], and (v) it can routinely be performed from as low input as $10 \mathrm{pg}$ of total RNA compared to about $100 \mathrm{ng}$ for microarrays and a double amplification protocol [63]. The latter is especially important, as the amount of RNA did not permit an analysis by GEP in approx. ten percent of patients in the GMMG-MM5 trial. Despite these advantages, there are also several caveats and challenges including data storage and handling due to the large size of RNAsequencing raw datasets, time-consuming bioinformatics analysis, and less standardization $[64,66,67]$. 


\section{Conclusion}

In conclusion, using an elaborated sampling, experimental and analysis strategy as reported here, we show for the first time that it is possible to prospectively perform and report molecular analyses in a randomized phase III multicenter trial in over $90 \%$ (iFISH) and $80 \%$ (GEP) of patients, respectively, within the first cycle of induction chemotherapy. Therefore, we validate that a trial strategy using either of the methods is possible, including reporting of potentially actionable targets. Risk assessment using our HM metascore allows to stratify patients with excellent/intermediate/adverse PFS and OS with survival rates of $98 \%$ vs. $68 \%$ vs. $25 \%$, respectively, after 5 years. In comparison to the rates by rISS of $86 \%$ vs. $65 \%$ vs. $40 \%$, respectively, both groups with better as well as more adverse survival are delineated by the HM metascore.

\section{Additional files}

Additional file 1: Table S1. PAM-based prediction error for light and heavy chain type as well as the sex of the patient. (PDF $1187 \mathrm{~kb}$ )

Additional file 2: Figure S1. Gene expression-based risk assessment as implemented in the GEP-R. Figure S2. Grouping myeloma into different subentities as implemented in the GEP-R. (PDF $1044 \mathrm{~kb}$ )

\section{Acknowledgements}

The authors thank Maria Dörner, Ewelina Nickel, and Birgit Schneiders for technical assistance in the enrichment of CD138-positive plasma cells, Tine Borowski, Michaela Brough, Michelle Ebentheuer, Marie-Christine Meffert, and Stephanie Pschowski-Zuck for technical assistance in iFISH, Véronique Pantesco for performing DNA-microarrays, Sybille Seyfried for assistance in patient documentation, as well as the Transcriptomics Platform at INSERM Montpellier.

\section{Authors' contributions}

$\mathrm{AS}$ and $\mathrm{DH}$ contributed to conception and design. UB gave administrative support. HS, MH, CS, KW, MSR, HG, and DH were responsible for the provision of study materials or patients. $\mathrm{DH}, \mathrm{SB}, \mathrm{ME}, \mathrm{UB}, \mathrm{TH}, \mathrm{CK}, \mathrm{AJ}, \mathrm{JM}$, and AS helped in the collection and assembly of data. AS, SB, and DH helped in the data analysis and interpretation, and manuscript writing. All authors read and approved the final manuscript.

\section{Funding}

This work was supported by the German Federal Ministry of Education (BMBF) "CAMPSIMM" (01ES1103).

\section{Availability of data and materials}

The dataset supporting the conclusions of this article is available in the ArrayExpress repository under accession number E-MTAB-2299.

\section{Ethics approval and consent to participate}

The GMMG-MM5 trial was approved by ethics committees of the University of Heidelberg and all participating sites. It was conducted according to the European Clinical Trial Directive (2005) and the Declaration of Helsinki.

\section{Consent for publication}

Not applicable.

\section{Competing interests}

The authors declare that they have no competing interest.

\section{Author details}

'Labor für Myelomforschung, Universitätsklinikum Heidelberg, Heidelberg, Germany. ${ }^{2}$ Medizinische Klinik V, Universitätsklinikum Heidelberg, Heidelberg,
Germany. ${ }^{3}$ Department of Internal Medicine II, Asklepios Klinik Altona, Hamburg, Germany. ${ }^{4}$ Deutsches Krebsforschungszentrum, Abteilung für Biostatistik, Heidelberg, Germany. ${ }^{5}$ Department I of Internal Medicine, University of Cologne, Cologne, Germany. ${ }^{6}$ Department of Internal Medicine III, Klinikum Chemnitz GmbH, Chemnitz, Germany. ${ }^{7}$ Department of Hematology, Oncology and Immunology, University of Tübingen, Tübingen, Germany. ${ }^{8}$ Nationales Centrum für Tumorerkrankungen, Heidelberg, Germany. ${ }^{9}$ Universität Heidelberg, Institut für Humangenetik, Heidelberg, Germany. ${ }^{10} \mathrm{IGH}$, CNRS, University of Montpellier, Montpellier, France. ${ }^{11}$ Department of Biological Hematology, CHU Montpellier, Montpellier, France.

Received: 29 March 2019 Accepted: 12 June 2019

Published online: 26 June 2019

References

1. Kyle RA, Rajkumar SV. Multiple myeloma. N Engl J Med. 2004;351:1860-73.

2. Sonneveld P, Schmidt-Wolf IG, van der Holt B, El Jarari L, Bertsch U, Salwender $\mathrm{H}$, et al. Bortezomib induction and maintenance treatment in patients with newly diagnosed multiple myeloma: results of the randomized phase III HOVON-65/ GMMG-HD4 trial. J Clin Oncol. 2012;30: 2946-55.

3. Hulin C, Belch A, Shustik C, Petrucci MT, Duhrsen U, Lu J, et al. Updated outcomes and impact of age with lenalidomide and low-dose dexamethasone or melphalan, prednisone, and thalidomide in the randomized, phase III FIRST Trial. J Clin Oncol. 2016:34:3609-17.

4. Lokhorst HM, Plesner T, Laubach JP, Nahi H, Gimsing P, Hansson M, et al. Targeting CD38 with daratumumab monotherapy in multiple myeloma. N Engl J Med. 2015;373:1207-19.

5. Child JA, Morgan GJ, Davies FE, Owen RG, Bell SE, Hawkins K, et al. Highdose chemotherapy with hematopoietic stem-cell rescue for multiple myeloma. N Engl J Med. 2003;348:1875-83.

6. Barlogie B, Mitchell A, van Rhee F, Epstein J, Morgan GJ, Crowley J. Curing myeloma at last: defining criteria and providing the evidence. Blood. 2014;124:3043-51.

7. Goldschmidt H, Lokhorst HM, Mai EK, van der Holt B, Blau IW, Zweegman S, et al. Bortezomib before and after high-dose therapy in myeloma: long-term results from the phase III HOVON-65/GMMG-HD4 trial. Leukemia. 2018:32:383-90.

8. Jethava Y, Mitchell A, Zangari M, Waheed S, Schinke C, Thanendrarajan S, et al. Dose-dense and less dose-intense total therapy 5 for gene expression profiling-defined high-risk multiple myeloma. Blood Cancer J. 2016;6:e471.

9. Kumar SK, Mikhael JR, Buadi FK, Dingli D, Dispenzieri A, Fonseca R, et al. Management of newly diagnosed symptomatic multiple myeloma: updated Mayo Stratification of Myeloma and Risk-Adapted Therapy (mSMART) consensus quidelines. Mayo Clin Proc. 2009:84:1095-110.

10. Palumbo A, Avet-Loiseau H, Oliva S, Lokhorst HM, Goldschmidt H, Rosinol L, et al. Revised international staging system for multiple myeloma: a report from International Myeloma Working Group. J Clin Oncol. 2015;33:2863-9.

11. Neben $K$, Jauch A, Bertsch U, Heiss C, Hielscher T, Seckinger A, et al. Combining information regarding chromosomal aberrations $t(4 ; 14)$ and del(17p13) with the International Staging System classification allows stratification of myeloma patients undergoing autologous stem cell transplantation. Haematologica. 2010;95:1150-7.

12. Avet-Loiseau H, Durie BG, Cavo M, Attal M, Gutierrez N, Haessler J, et al. Combining fluorescent in situ hybridization data with ISS staging improves risk assessment in myeloma: an International Myeloma Working Group collaborative project. Leukemia. 2013;27:711-7.

13. Hose D, Reme T, Hielscher T, Moreaux J, Meissner T, Seckinger A, et al. Proliferation is a central independent prognostic factor and target for personalized and risk adapted treatment in multiple myeloma. Haematologica. 2011;96:87-95.

14. Shaughnessy JD, Zhan F, Burington BE, Huang Y, Colla S, Hanamura I, et al. A validated gene expression model of high-risk multiple myeloma is defined by deregulated expression of genes mapping to chromosome 1 . Blood. 2007;109:2276-84.

15. Decaux O, Lodé L, Magrangeas F, Charbonnel C, Gouraud W, Jézéquel P, et al. Prediction of survival in multiple myeloma based on gene expression profiles reveals cell cycle and chromosomal instability signatures in high-risk patients and hyperdiploid signatures in low-risk patients: a study of the Intergroupe Francophone du Myélome. J Clin Oncol. 2008;26:4798-805.

16. Reme $T$, Hose $D$, Theillet $C$, Klein B. Modeling risk stratification in human cancer. Bioinformatics. 2013;29:1149-57. 
17. Kuiper R, Broyl A, de Knegt $Y$, van Vliet $M H$, van Beers EH, van der Holt B, et al. A gene expression signature for high-risk multiple myeloma. Leukemia. 2012;26:2406-13.

18. Chng WJ, Chung TH, Kumar S, Usmani S, Munshi N, Avet-Loiseau H, et al. Gene signature combinations improve prognostic stratification of multiple myeloma patients. Leukemia. 2016;30:1071-8.

19. Chng WJ, Dispenzieri A, Chim CS, Fonseca R, Goldschmidt H, Lentzsch S, et al. IMWG consensus on risk stratification in multiple myeloma. Leukemia. 2014;28:269-77.

20. Fonseca R, Bergsagel PL, Drach J, Shaughnessy J, Gutierrez N, Stewart AK, et al. International Myeloma Working Group molecular classification of multiple myeloma: spotlight review. Leukemia. 2009;23:2210-21.

21. Hose D, Rème T, Meissner T, Moreaux J, Seckinger A, Lewis J, et al. Inhibition of aurora kinases for tailored risk-adapted treatment of multiple myeloma. Blood. 2009;113:4331-40.

22. Sprynski AC, Hose D, Caillot L, Reme T, Shaughnessy JD Jr, Barlogie B, et al. The role of IGF-1 as a major growth factor for myeloma cell lines and the prognostic relevance of the expression of its receptor. Blood. 2009;113:4614-26.

23. Jethava YS, Mitchell A, Epstein J, Zangari M, Yaccoby S, Tian E, et al. Adverse metaphase cytogenetics can be overcome by adding bortezomib and thalidomide to fractionated melphalan transplants. Clin Cancer Res. 2017;23:2665-72.

24. Neben $\mathrm{K}$, Lokhorst HM, Jauch A, Bertsch U, Hielscher T, van der Holt B, et al. Administration of bortezomib before and after autologous stem cell transplantation improves outcome in multiple myeloma patients with deletion 17p. Blood. 2012;119:940-8.

25. Perrot A, Lauwers-Cances V, Tournay E, Hulin C, Chretien ML, Royer B, et al. Development and validation of a cytogenetic prognostic index predicting survival in multiple myeloma. J Clin Oncol. 2019:JCO1800776.

26. Hofste op Bruinink D, van Duin M, Beksac M, Driessen C, Ludwig H, Vermeulen M, et al. Differential effect of upfront intensification treatment in genetically defined myeloma risk groups - a combined analysis of ISS, Del17p and SKY92 Scores in the EMN-02/HOVON-95 MM Trial. Blood. 2018;132:3186.

27. Sherborne AL, Shah V, Ellis S, Begum F, Kendall J, Johnson DC, et al. Improving outcomes for patients with high-risk myeloma via prospective trial evidence: The Myeloma UK <em>Nine Optimum</em> Trial. Blood. 2017;130:1767

28. Van Beers EH, Terragna C, Martello M, Zamagni E, Cavo M, van Vliet MH, et al Mmprofiler with SKY92 Combined with ISS Identifies High and Low Risk Multiple Myeloma in the VTD Arm of Gimema-MMY-3006. Blood. 2017;130:4358.

29. Mai EK, Bertsch U, Durig J, Kunz C, Haenel M, Blau IW, et al. Phase III trial of bortezomib, cyclophosphamide and dexamethasone (VCD) versus bortezomib, doxorubicin and dexamethasone (PAd) in newly diagnosed myeloma. Leukemia. 2015:29:1721-9.

30. Hose D, Moreaux J, Meissner T, Seckinger A, Goldschmidt H, Benner A, et al. Induction of angiogenesis by normal and malignant plasma cells. Blood. 2009:114:128-43.

31. Meißner T, Seckinger A, Rème T, Hielscher T, Möhler T, Neben K, et al. Gene expression profiling in multiple myeloma-reporting of entities, risk, and targets in clinical routine. Clin Cancer Res. 2011;17:7240-7.

32. Seckinger A, Delgado JA, Moser S, Moreno L, Neuber B, Grab A, et al. Target expression, generation, preclinical activity, and Pharmacokinetics of the BCMA-T cell bispecific antibody EM801 for multiple myeloma treatment. Cancer Cell. 2017:31:396-410.

33. Seckinger A, Meißner T, Moreaux J, Depeweg D, Hillengass J, Hose K, et al. Clinical and prognostic role of annexin A2 in multiple myeloma. Blood. 2012;120:1087-94

34. Seckinger A, Meissner T, Moreaux J, Goldschmidt H, Fuhler GM, Benner A, et al. Bone morphogenic protein 6: a member of a novel class of prognostic factors expressed by normal and malignant plasma cells inhibiting proliferation and angiogenesis. Oncogene. 2009;28:3866-79.

35. Seckinger A, Hillengass J, Emde $M$, Beck S, Kimmich $C$, Dittrich $T$, et al CD38 as Immunotherapeutic target in light chain amyloidosis and multiple myeloma-association with molecular entities, risk, survival, and mechanisms of upfront resistance. Frontiers in Immunology. 2018;9.

36. Neben $\mathrm{K}$, Jauch A, Hielscher T, Hillengass J, Lehners N, Seckinger A, et al. Progression in smoldering myeloma is independently determined by the chromosomal abnormalities del(17p), t(4;14), Gain 1q, Hyperdiploidy, and Tumor Load. J Clin Oncol. 2013;31:4325-32.

37. R-Development-Core-Team. R: A language and environment for statistical computing. Vienna: R Foundation for Statistical Computing; 2008.
38. Gentleman RC, Carey VJ, Bates DM, Bolstad B, Dettling M, Dudoit S, et al Bioconductor: open software development for computational biology and bioinformatics. Genome Biol. 2004;5:R80.

39. Bergsagel PL, Kuehl WM, Zhan F, Sawyer J, Barlogie B, Shaughnessy J. Cyclin $D$ dysregulation: an early and unifying pathogenic event in multiple myeloma. Blood. 2005;106:296-303.

40. Hose D, Rossi J-F, Ittrich C, DeVos J, RŠme T, Benner A, et al. Molecular classification of multiple myeloma (MM) based on gene expression profiling (GEP) and fluorescence in situ hybridisation (FISH) is an independent predictor for event free survival (EFS). [abstract]. Blood. 2005;106:150a-a.

41. Zhan F, Huang Y, Colla S, Stewart JP, Hanamura I, Gupta S, et al. The molecular classification of multiple myeloma. Blood. 2006;108:2020-8.

42. Tibshirani R, Hastie T, Narasimhan B, Chu G. Diagnosis of multiple cancer types by shrunken centroids of gene expression. Proc Natl Acad Sci U S A 2002;99:6567-72.

43. Therneau TM, Grambsch PM. Modeling survival data: extending the Cox model. Modeling Survival Data: Extending the Cox Model: Springer-Verlag, New York; 2000. p. XIV, 350.

44. Andersen PK, Gill RD. Cox's Regression Model for Counting Processes: A Large Sample Study. Annals of Statistics. 1982;10:1100-20.

45. Fleming TR, Harrington DP. Nonparametric estimation of the survival distribution in censored data. Comm in Stat Theory and Methods. 1984;13:2469-86.

46. Kaplan EL, Meier P. Nonparametric estimation from incomplete observations. Journal of the American Statistical Association. 1958:53:457-81.

47. Harrington DP, Fleming TR. A class of rank test procedures for censored survival data. Biometrika. 1982;69:553-66.

48. Schumacher M, Graf E, Gerds T. How to assess prognostic models for survival data: a case study in oncology. Methods Inf Med. 2003;42:564-71.

49. Mogensen UB, Ishwaran H, Gerds TA. Evaluating random forests for survival analysis using prediction error curves. Journal of statistical software. 2012;50:1-23.

50. van de Wiel MA, Berkhof J, van Wieringen WN. Testing the prediction error difference between 2 predictors. Biostatistics. 2009;10:550-60.

51. Nilsson T, Hoglund M, Lenhoff S, Rylander L, Turesson I, Westin J, et al. A pooled analysis of karyotypic patterns, breakpoints and imbalances in 783 cytogenetically abnormal multiple myelomas reveals frequently involved chromosome segments as well as significant age- and sex-related differences. Br J Haematol. 2003:120:960-9.

52. Cavo M, Beksac M, Dimopoulos MA, Pantani L, Gay F, Hájek R, et al. Intensification therapy with bortezomib-melphalan-prednisone versus autologous stem cell transplantation for newly diagnosed multiple myeloma: an intergroup, multicenter, phase III study of the European Myeloma Network (EMN02/H095 MM Trial). Blood. 2016;128:673.

53. Attal M, Lauwers-Cances V, Hulin C, Leleu X, Caillot D, Escoffre M, et al. Lenalidomide, bortezomib, and dexamethasone with transplantation for myeloma. N Engl J Med. 2017;376:1311-20.

54. Einsele H, Engelhardt M, Tapprich C, Muller J, Liebisch P, Langer C, et al. Phase II study of bortezomib, cyclophosphamide and dexamethasone as induction therapy in multiple myeloma: DSMM XI trial. Br J Haematol. 2017:179:586-97.

55. Durie BG, Hoering A, Abidi MH, Rajkumar SV, Epstein J, Kahanic SP, et al. Bortezomib with lenalidomide and dexamethasone versus lenalidomide and dexamethasone alone in patients with newly diagnosed myeloma without intent for immediate autologous stem-cell transplant (SWOG S0777): a randomised, open-label, phase 3 trial. Lancet. 2017:389:519-27.

56. Lahuerta JJ, Paiva B, Vidriales MB, Cordon L, Cedena MT, Puig N, et al. Depth of Response in Multiple Myeloma: A Pooled Analysis of Three PETHEMA/ GEM Clinical Trials. J Clin Oncol. 2017;35:2900-10.

57. Moreau P, Cavallo F, Leleu X, Hulin C, Amiot M, Descamps G, et al. Phase I study of the anti insulin-like growth factor 1 receptor (IGF-1R) monoclonal antibody, AVE1642, as single agent and in combination with bortezomib in patients with relapsed multiple myeloma. Leukemia. 2011;25:872-4.

58. Trudel S, Li ZH, Wei E, Wiesmann M, Chang H, Chen C, et al. CHIR-258, a novel, multitargeted tyrosine kinase inhibitor for the potential treatment of $\mathrm{t}(4 ; 14)$ multiple myeloma. Blood. 2005;105:2941-8.

59. Sprynski AC, Hose D, Kassambara A, Vincent $L$, Jourdan M, Rossi JF, et al. Insulin is a potent myeloma cell growth factor through insulin/IGF-1 hybrid receptor activation. Leukemia. 2010;24:1940-50.

60. Kuiper $R$, van Duin M, van Vliet MH, Broij A, van der Holt B, El Jarari L, et al. Prediction of high- and low-risk multiple myeloma based on gene expression and the International Staging System. Blood. 2015;126:1996-2004. 
61. Sherborne AL, Begum DB, Price A, Johnson DC, Ellis S, Smith C, et al. Identifying ultra-high risk myeloma by integrated molecular genetic and gene expression profiling. Blood. 2016;128:4407.

62. Zhou Y, Zhang Q, Stephens O, Heuck CJ, Tian E, Sawyer JR, et al. Prediction of cytogenetic abnormalities with gene expression profiles. Blood. 2012;119: e148-e150.

63. Seckinger A, Bähr-Ivacevic T, Benes V, Hose D. RNA-Sequencing from LowInput Material in Multiple Myeloma for Application in Clinical Routine. In: Heuck C, Weinhold, N., editor. Multiple Myeloma - Methods and Protocols. Methods Molecular Biology: Humana Press; 2018

64. Xu J, Thakkar S, Gong B, Tong W. The FDA's experience with emerging genomics technologies-past, present, and future. AAPS J. 2016;18:814-8.

65. Seckinger A, Meissner T, Moreaux J, Benes V, Hillengass J, Castoldi M, et al. miRNAs in multiple myeloma-a survival relevant complex regulator of gene expression. Oncotarget. 2015:6:39165-83.

66. Conesa A, Madrigal P, Tarazona S, Gomez-Cabrero D, Cervera A, McPherson A, et al. A survey of best practices for RNA-seq data analysis. Genome Biol. 2016;17:13.

67. Costa-Silva J, Domingues D, Lopes FM. RNA-Seq differential expression analysis: an extended review and a software tool. PLoS One. 2017;12: e0190152.

\section{Publisher's Note}

Springer Nature remains neutral with regard to jurisdictional claims in published maps and institutional affiliations.

Ready to submit your research? Choose BMC and benefit from:

- fast, convenient online submission

- thorough peer review by experienced researchers in your field

- rapid publication on acceptance

- support for research data, including large and complex data types

- gold Open Access which fosters wider collaboration and increased citations

- maximum visibility for your research: over $100 \mathrm{M}$ website views per year

At $\mathrm{BMC}$, research is always in progress.

Learn more biomedcentral.com/submissions 